\title{
Necrólise epidérmica tóxica secundária ao uso da citosina-arabinosídeo em dose intermediária
}

\author{
M.S. Figueiredo, M. Yamamoto, J . Kerbauy \\ Disciplina de Hematologia e Hemoterapia do Departamento de Medicina da Universidade Federal de São Paulo — Escola Paulista de \\ Medicina, São Paulo, SP.
}

RESUMO - A necrólise epidérmica tóxica é afecção dermatológica secundária ao uso de drogas e corresponde à síndrome de Lyell, relacionada ao eritema multiforme e à síndrome de StevensJ ohnson.

Овј етіvos. Relatar um caso de necrólise epidérmica fatal secundária à citosina-arabinosídeo (Ara-C) em dose intermediária.

Relato de caso. Paciente do sexo feminino, com 16 anos de idade, portadora de leucemia linfóide aguda - LLA-L1. Iniciou tratamento segundo o protocolo do Grupo Brasileiro de Tratamento da Leucemia Infantil/85, alto risco. $\mathrm{Na}$ fase II da indução, após o uso de Ara-C na dose de $1,5 \mathrm{~g} / \mathrm{m}^{2}$, intravenoso, 12/12h $x$ três dias, desenvolveu múl-

\section{INTRODUÇÃO}

A citosina-arabinosídeo (Ara-C) é um análogo da pirimidina, que inibe competitivamente a DNApol imerase, afetando a fase $\mathrm{S}$ do ciclo celular. Foi introduzida no tratamento do câncer, em 1964, e não só sua eficácia terapêutica, como também sua toxi cidade, é estritamente rel acionada ao esquema de administraçãoํ. A partir de 1979, vários ensai os clínicos foram realizados com o propósito de avaliar o uso de Ara-C em altas doses, demonstrando sua atividade em diferentes tipos de tumores sólidos, além de leucemias agudas refratárias. Várias manifestações de toxicidade dessa droga têm sido descritas, sendo as mais freqüentes as do sistema nervoso central, do sistema ocular e as dermatológicas ${ }^{2}$. O objetivo do presente trabalho é o de relatar um caso de necrólise epidérmica fatal secundária ao Ara-C, situação esta ainda não referida na literatura.

\section{APRE SENTAÇÃO DO CASO}

Paciente de 16 anos, feminina, branca, brasileira, procurou o Serviço de H ematologia e Hemoterapia da UNIFESP/EPM com história de escurecimento da visão e perda de consciência há cinco dias. Ao tiplas lesões cutâneas bolhosas, que aumentaram rapidamente por progressão das bordas. As boIhas continham secreção serosa, evoluíram para ul ceração superficial central, com infecção secundária múltipla. Faleceu por septicemia, no $13^{\circ}$ dia após o início do quadro dermatológico.

Conclusão. O Ara-C tem sido relacionado a diversas manifestações de toxicidade dermatológica; no entanto, até o momento, não há relato de necrólise epidérmica tóxica, sendo este o primeiro caso da literatura.

UNITERMOS: Citosina-arabinosídeo - efeitos adversos. Doenças da pele vésico-bolhosas. Eritema acral secundário à quimi oterapia.

exame físico, apresentava descoramento de mucosas e equimoses de membros inferiores. Hemograma inicial revel ou $\mathrm{Hb}=7,0 \mathrm{~g} / \mathrm{dL} ; \mathrm{Ht}=20 \% ; \mathrm{GB}=$ $54.300 / \mathrm{mm}^{3} ; 92 \%$ de blastos linfói des e pl aquetas $=$ $25.000 / \mathrm{mm}^{3}$. N o miel ograma, $90 \%$ de células nucleares eram linfoblastos, escore +2 , compatível com leucemia linfóide aguda L1 (LLA-L1). O PAS encontrava-se positivo em $49 \%$ dos blastos. A imunofenotipagem linfocitária mostrou $13 \%$ de positividade para linfócitos T e 3\% para linfócitos B (LLA não $T$ não $B$ ). A pesquisa de células blásticas no líquor cefalorraquidiano mostrou-se positiva. Foi ini ciado tratamento qui mi oterápi co segundo o protocolo do Grupo Brasileiro de Tratamento de Leucemias da I nfância/85, alto risco (GBTLI-85-AR).

A primeira fase da indução transcorreu sem complicações. No 36 dia da indução, iniciou-se a administração de Ara-C na dose de $1,5 \mathrm{~g} / \mathrm{m}^{2}$ de superfície corpórea, intravenosa, a cada 12 horas, por três dias. No segundo dia após o início da infusão, observou-se lesão bol hosa de cerca de $1 \mathrm{~cm}$ de diâmetro, na região glútea (fig. 1), que progrediu rapidamente de tamanho, com liberação de conteúdo cristalino e necrose central (fig. 2). Concomitantemente, apareceram outras lesões exoulceradas com evolução para crostosas, de vários tamanhos, dolorosas, nas axilas, coxas, pescoço e 
Fig. 1 - Lesão bolhosa inicial.

face. Nessa ocasião, o hemograma revel ou intensa neutropenia ( $\mathrm{GB}=400 / \mathrm{mm}^{3}$, com $100 \%$ de linfócitos). A paciente desenvolveu infecção secundária múltipla por S. aureus, Klebsi ella sp. e Enterobacter. Apesar da antibioticoterapia instituída, veio a falecer por septicemia no 13 dia após o início do quadro dermatológico.

\section{DISCUSSÃO}

Diversas são as manifestações tóxicas descritas em associação ao uso de Ara-C em alta dose. Em estudo recente, Graves \& Hooks ${ }^{3}$ observaram incidência de $37,7 \%$ de toxicidade do sistema nervoso central , 37,7\% de toxicidade ocular e 45,3 de toxicidade dermatológica. Outros efeitos adversos, observados em menor freqüência, incluem falência pulmonar subaguda ${ }^{4}$, disfunção hepática e icterícia5, além da "síndrome da Citarabina", caracterizada por febre, mialgia, dores ósseas e, ocasionalmente, dor torácica, "rash cutâneo" maculopapular e conjuntivite ${ }^{1}$.

O quadro dermatológi co mais freqüente éo eritema acral induzido por quimioterapia (eritema palmo-plantar, síndrome de Lokich-Moore ou eritrodisestesia palmo-plantar), uma eritrodermia dolorosa, macular, intensa, em palma das mãos e dedos, autolimitada6. São descritos vários tipos de eritema acral induzi do por quimioterapia, incluindo-se casos que evoluem com bol has e posterior descamação. O eritema acral parece estar associado a diversos agentes terapêuticos, embora não haja relato de formas bolhosas em pacientes que não utilizaram o Ara-C, além desta droga ter sido relacionada como agente único em $55 \%$ dos casos de

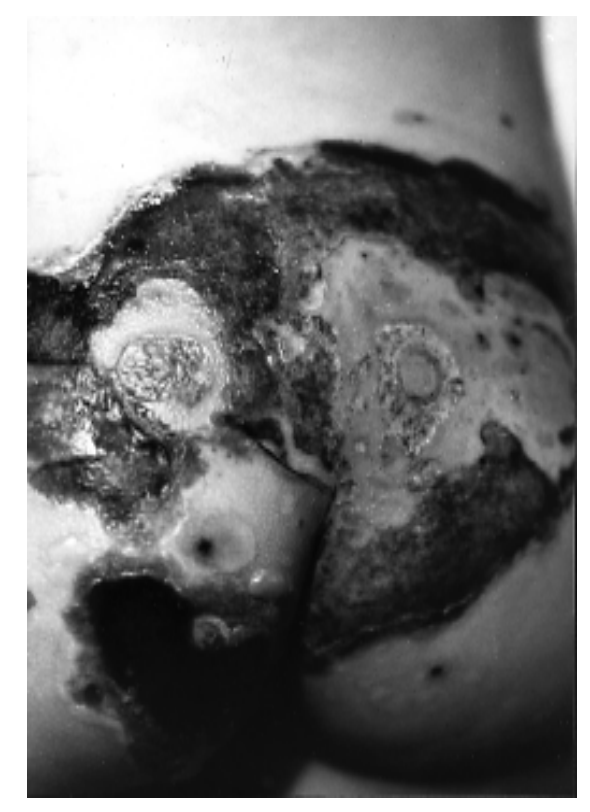

Fig. 2 - Lesão em nádegas, no 10 o dia de evol ução, evidenciando a grande progressão e a área necrótica central.

eritema acral bolhoso 5 . O diagnóstico diferencial do eritema acral induzido por quimi oterapia é feito com eritema multiforme e outras reações a drogas.

A hidroadenite écrina neutrofílica é outra dermatopatia relacionada a diversos agentes quimioterápi cos e, principal mente, ao Ara-C, e corresponde à necrose de glândulas écrinas, com infiltrado neutrofílico, formando nódulos endurecidos com área necrótica central ${ }^{7}$.

No presente caso, o quadro dermatológico corresponde à síndrome de Lyell ou necróli se epi dérmica tóxica, relacionada ao eritema mul ti forme è síndrome de Stevens-J ohnson, que se caracteriza pela formação de vesículas ou bolhas grandes e flácidas, com separação entre epiderme e derme e posterior descamação, semelhante à queimadura de segundo grau, freqüentemente associada ao uso de medicamentos ${ }^{8}$. E mbora o uso de citosina-arabinosídeo esteja relacionado a diversas manifestações de toxicidade dermatol ógica, não foi relatada, até o momento, necrólise epidérmica tóxica associada ao uso dessa droga, justifi cando a publicação do caso presente.

\section{SUMMARY}

\section{Toxic epidermal necrolysis as adverse effect of intermediate dose of cytosine arabinoside}

Toxic epidermal necrolysis is a drug-induced dermatologic disease related to Lyell syndrome, erythema multiforme and Stevens-J ohnson syndrome.

Purpose. To report a fatal case of toxic epidermal necrolysis owing to intermediate dose of cytarabine. 
CASE REPORT. A 16 year-old female patient with acute Iymphocytic leukemia (LLA-L1) treated with theProtocol of the Brazilian Group for Treatment of Leukemia of Childwood (GBTLI-85-AR). On the second day after the administration of intermediate dose of cytarabine (1.5g/ $\mathrm{m}^{2}$ IV every 12 hours for 3 days), she presented bullous lesions in the left buttock that disseminated envolving to necrosis, sepsis, and death on the $13^{\text {th }}$ day.

Conclusion. Cytarabine is frequently associated with dermatologic toxicity but, until now, thereis no other case of toxic epidermal necrolysis described. [Rev Ass Med Brasil 1998; 44(1): 53-5.]

KEY WORDS: Cytarabine - adverse effect. Skin disease vesiculobullous. Toxic epidermal necrolysis.

\section{REFERÊ NCIAS BIBLIOGRÁFICAS}

1. Castleberry RP, Crist WM, Holbrook T, Malluh A, Gaddy D. The cytosine arabinoside (Ara-C) syndrome. Med Pediatr
Oncol 1981; 9: 257-64

2. Barnett MJ , Richards MA, Ganesan TS et al. Central nervous system toxicity of high-dose cytosine arabinoside. Sem Oncol 1985; 12(suppl 3): 23-8.

3. Graves T, Hooks MA. Drug-induced toxicities associated with high-dose cytosine arabinoside infusions. Pharmacotherapy 1989; 9: 23-8.

4. Anderson BS, Cogan BM, Keating MJ et al. Subacute pulmonary failure complicating therapy with high-dose araC in acute leukemia. Cancer 1985; 56: 2.181-4.

5. Waltzer J F, Flowers FP. Bullous variant of chemotherapyinduced acral eryhtema (letter). Arch Dermatol 1993; 129: 43-5.

6. Baack BR, Burgdoff WHC. Chemotherapy-induced acral erythema. J Am Acad Dermatol 1991; 24: 457-61.

7. Flynn TC, Harrist TJ, Murphy GF, Loss RW, Moschella SL. Neutrofilic eccrine hidradenitis: a distinctive rash associated with cytarabine therapy and acute leukemia. J Am Acad Dermatol 1984; 11: 584-90.

8. Bastuji-Garin S, Razany B, Stern RS et al. Clinical classification of cases of toxic epidermal necrolysis, StevensJ ohnson syndromes, and erythema multiforme. Arch Dermatol 1993; 129: 92-6. 